\title{
Brivaracetam Retention Rate and Seizure Outcomes in Patients with Drug-Resistant Focal Epilepsy Included in the Medical Need Program in Belgium: A Real-World, Multicenter, Chart Review
}

\author{
Chantal Depondt $^{1} \cdot$ Wim Van Paesschen ${ }^{2}$ D $\cdot$ Kenou van Rijckevorsel ${ }^{3} \cdot$ Iryna Leunikava $^{4} \cdot$ France Ferrière $^{5}$ (D)
}

Accepted: 30 March 2021 / Published online: 31 May 2021

(c) The Author(s) 2021

\begin{abstract}
Background New treatments are needed for patients with drug-resistant epilepsy to improve seizure control without decreasing quality of life.

Objective In Belgium, a Medical Need Program (MNP) was initiated to make a new antiepileptic drug (brivaracetam; highaffinity synaptic vesicle protein $2 \mathrm{~A}$ ligand) available as adjunctive therapy to treat focal seizures in patients failing treatment with three or more different antiepileptic drugs. This is a real-world chart review of the majority of patients (71\%) enrolled in the MNP.

Patients and Methods Retention and seizure outcomes of brivaracetam adjunctive treatment were evaluated in 175 patients aged $\geq 16$ years enrolled in the MNP between June 2016 and May 2017 at six centers; 95.4\% were previously/concomitantly treated with levetiracetam. Safety events data were also collected.

Results In this highly drug-resistant population, $85.8 \%, 73.9 \%$, and $64.9 \%$ of patients remained on brivaracetam, while seizure frequency decreased from baseline in $32.0 \%, 37.1 \%$, and $37.3 \%$ of patients after 3, 6, and 9 months' treatment, respectively. Patients achieving 3-month seizure freedom increased from $3.2 \%$ after 3 months' treatment to $10.2 \%$ and $10.7 \%$ after 6 and 9 months' treatment, respectively. Six-month seizure freedom was achieved by $5.7 \%$ of patients at any time. Qualitative evaluation of seizures by physicians demonstrated $44.2 \%, 38.8 \%$, and $43.2 \%$ of patients improved and $42.8 \%$, $50.9 \%$, and $50.6 \%$ remained unchanged during 3, 6, and 9 months' follow-up, respectively. No safety signals were identified. Conclusions Retention was high during 9 months of brivaracetam treatment in drug-resistant patients, including those previously/concomitantly treated with levetiracetam; 3-month seizure freedom increased from $3.2 \%$ after 3 months to $10.7 \%$ after 9 months of treatment.
\end{abstract}

\section{Introduction}

France Ferrière

france.ferriere@ucb.com

1 Reference Center for the Treatment of Refractory Epilepsy, Hôpital Erasme, Université Libre de Bruxelles, Route de Lennik 808, 1070 Brussels, Belgium

2 Refractory Epilepsy Center, University Hospitals Leuven, Herestraat 49, 3000 Leuven, Belgium

3 Centre Hospitalier Neurologique William Lennox, Allée de Clerlande 6, 1340 Ottignies, Belgium

4 UCB Pharma, Alfred-Nobel-Straße 10, 40789 Monheim am Rhein, Germany

5 UCB Pharma, Allée de la Recherche 60, 1070 Brussels, Belgium
Approximately one-third of epilepsy patients develop drug resistance, defined as "failure of adequate trials of two tolerated and appropriately chosen and used antiepileptic drug (AED) schedules (as monotherapies or in combination) to achieve sustained seizure freedom" [1,2]. Moreover, about a half of treated patients experience mild to moderately severe adverse reactions $[3,4]$. Therefore, new treatments are needed for patients with drug-resistant epilepsy to improve seizure control without decreasing their quality of life.

In this context, brivaracetam, a synaptic vesicle protein 2A (SV2A) ligand differing from levetiracetam by its rapid brain penetration, lack of direct effect on AMPA receptors, and higher affinity for SV2A, was developed $[5,6]$. 


\section{Key Points}

In patients with drug-resistant epilepsy, retention rate was high after brivaracetam treatment initiation $(85.8 \%$ after 3 months, $73.9 \%$ after 6 months, and $64.9 \%$ after 9 months).

Seizure frequency decreased from baseline in $32.0 \%$, $37.1 \%$, and $37.3 \%$ of patients after 3,6 , and 9 months treatment, respectively.

The percentage of patients with 3-month seizure freedom increased from $3.2 \%$ after 3 months' treatment to $10.2 \%$ and $10.7 \%$ after 6 and 9 months' treatment, respectively.

Qualitative seizure improvements were reported in $61 / 138,45 / 116$, and $35 / 81$ of patients after 3,6 , and 9 months, respectively.

Brivaracetam showed higher potency and efficacy as an anti-seizure and antiepileptogenic agent than levetiracetam in preclinical epilepsy models [7, 8]. Clinical trials have shown that brivaracetam used as an adjunctive therapy reduced seizure frequency and had a favorable safety profile [9]. Moreover, brivaracetam was associated with fewer behavioral adverse events than levetiracetam [10]. In the European Union, brivaracetam is approved as adjunctive therapy in the treatment of focal seizures with or without secondary generalization (new terminology of the International League Against Epilepsy: evolution to bilateral tonicclonic seizures) in epilepsy patients from the age of 4 years [11-13].

In Belgium, a Medical Need Program (MNP) was initiated as an early access program to make brivaracetam available before commercialization for adolescents and adults with epilepsy who could benefit from this AED as adjunctive therapy in the treatment of focal seizures [14]. To meet the Belgian eligibility criteria for reimbursement of the most recently licensed AEDs, including brivaracetam, lacosamide, and perampanel, all patients included in the MNP had failed treatment with three or more different AEDs due to a lack of efficacy or occurrence of unacceptable adverse events [15]. Here, we present the results of a real-world chart review based on data from the MNP, which primarily evaluated brivaracetam retention rates after 3, 6, and 9 months of treatment. Additional data collected were seizure frequency and qualitative clinical seizure evaluation after 3, 6, and 9 months of treatment, and changes in AEDs. Safety information was collected as additional data and analyzed post hoc.

\section{Methods}

\subsection{Design and Population}

This retrospective, longitudinal, multicenter chart review was based on data from the MNP collected in six centers, representing $71 \%$ of the total MNP population: Universitair Ziekenhuis Leuven (Leuven), Centrum voor Epilepsie en Psycho-Organische Stoornissen (Duffel), Hôpital Erasme (Brussels), Cliniques Universitaires Saint-Luc (Brussels), Centre Hospitalier Universitaire de Liège (Liège), and Centre Hospitalier Neurologique William Lennox (Ottignies-Louvain-la-Neuve).

Eligible patients were enrolled in the MNP to initiate brivaracetam treatment between 10 June 2016 and 31 May 2017. All patients were $\geq 16$ years of age, had uncontrolled focal seizures despite previous treatments with three or more AEDs (in monotherapy or adjunctive therapy, concomitant or life-time used; due to therapeutic failure or adverse events), of which one was levetiracetam (except when levetiracetam was not considered appropriate in the opinion of the treating physician).

The Ethics Committees of all participating centers were approached for their consent of the MNP and to inform them of the data collection.

\subsection{Data Collection and Analysis}

Data were collected from patients' records and anonymized by either the treating physicians or designated personnel. Data entry was conducted by site personnel or data entry assistants upon request of the treating physicians and approval by Ethics Committees.

At baseline (day of brivaracetam initiation), year of birth, gender, lifetime history of AEDs prescribed before brivaracetam, non-drug treatment (e.g., epilepsy surgery, vagus nerve stimulation, deep brain stimulation), monthly seizure frequency during 6 months before brivaracetam initiation, brivaracetam initiation date, and AEDs concomitant to brivaracetam, were collected. At 3, 6, and 9 months after brivaracetam initiation, the monthly seizure frequency, qualitative clinical seizure evaluation (changes in seizure frequency according to routine clinical physician's assessment), treatment continuation/discontinuation status, and treatment end date, if applicable, were collected. Monthly seizure frequency was collected and analyzed in categories to ease the evaluation of disease evolution, as follows: (1) seizure-free, (2) < 6 per month, (3) 6-15 per month, (4) 16-30 per month, and (5) > 30 per month. At last followup, the numbers of lifetime AEDs and AEDs concomitant to brivaracetam were collected. 
Safety information was not initially the focus of this chart review but was collected during the MNP, based on a spontaneous reporting system. As such, safety events were analyzed post hoc. Psychiatric, central nervous system-related, and other treatment-emergent safety events were reported to UCB Patient Safety by the treating physicians.

\subsection{Statistical Analysis}

The number of patients analyzed was based on the number of patients included in the MNP in the six participating centers. Analyses were performed on the entire chart review population and the subpopulation of patients on concomitant levetiracetam at last follow-up.

Statistical analyses were descriptive (mean, standard deviation (SD), median, and range for quantitative variables; frequencies for categorical variables). Retention rates were calculated as proportion of patients maintaining brivaracetam at 3, 6, and 9 months and using Kaplan-Meier estimates. The number of censored observations, i.e., last observation for each patient after which no data were available, was recorded at baseline, and at 3,6, and 9 months. No correction methods were used to complete missing values. Percentages of seizure-free patients were calculated during the first 3 months, between 3 and 6 months, and between 6 and 9 months after brivaracetam initiation. Statistical analyses were performed using a proprietary macro-enriched Excel file (XDUstat v1.3), which was run under Microsoft Excel 2016 MSO 32-bit.

\section{Results}

\subsection{Demographic Characteristics of the Population}

Data from 178 patients were collected. Three patients were excluded from all analyses: two due to off-label brivaracetam use (one treated for progressive myoclonic epilepsy and one treated with brivaracetam as monotherapy) and one due to unknown brivaracetam initiation date (Fig. 1). After 3, 6, and 9 months of treatment, data from 169, 161, and 131 patients were included in analyses, respectively. After 9 months, data were no longer available for 44 patients due to absence of follow-up data or patient lost to follow-up ( $n$ $=23$ ) or patients having a shorter observation period due to later enrollment $(n=21)$ (Fig. 1). In the latter 21 patients, the observation period was less than 9 months because patients were enrolled between June 2016 and May 2017,

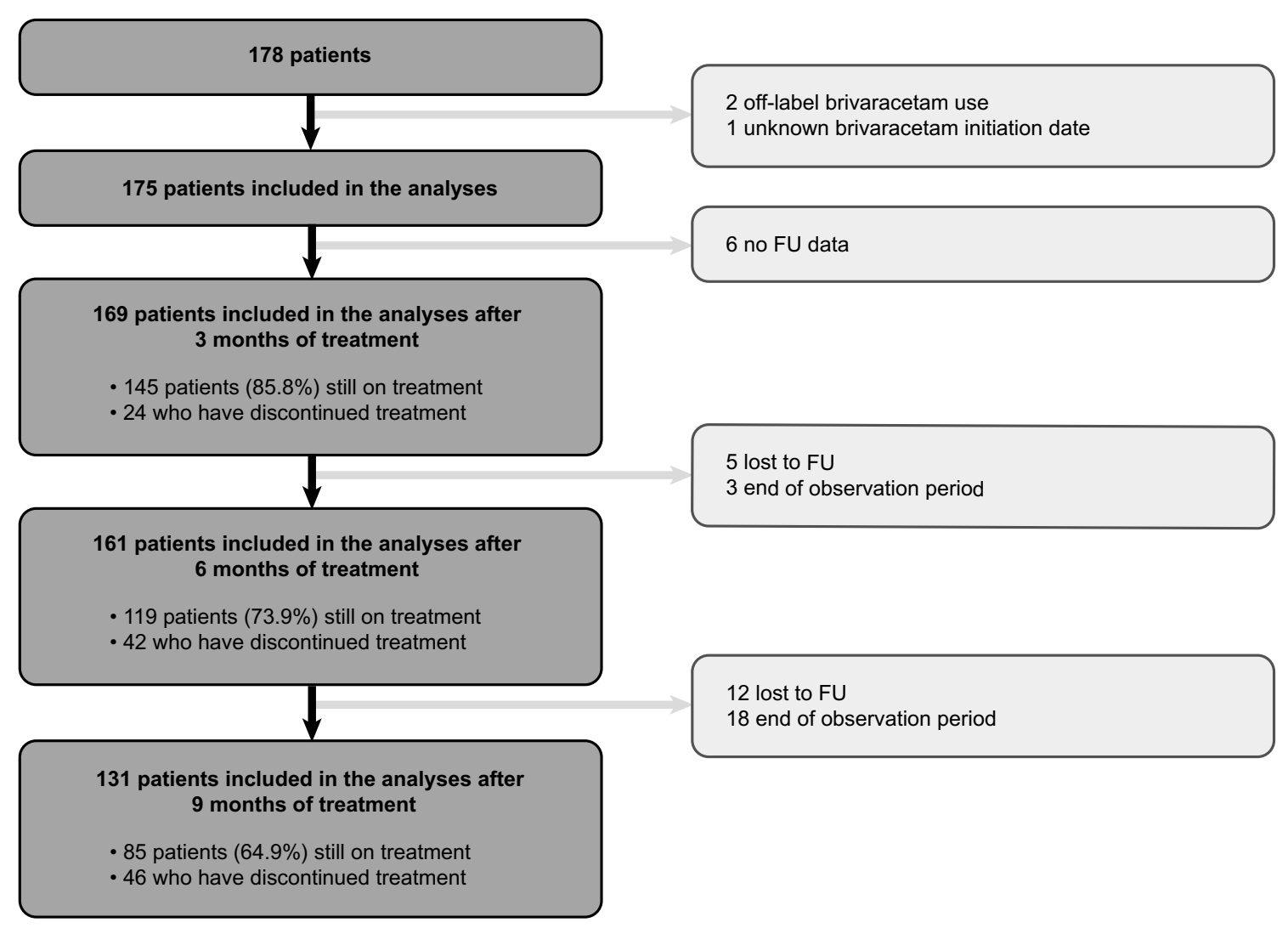

Fig. 1 The STROBE flow chart. $F U$ follow-up 
and data were collected between October 2017 and November 2017. Patients enrolled towards the end of the inclusion period therefore received brivaracetam for less than 9 months at the time of data collection (end of their observation period). The mean follow-up duration was 9 months (SD 1.5 months), starting at the inclusion in the MNP.

The median age of patients was 37 years (range 16-71 years) and $54.9 \%$ were female (Table 1). Patients had highly drug-resistant epilepsy as reflected by the 85 patients (48.6\%) who had used non-drug treatments (mainly vagus nerve stimulation (72 patients) and epilepsy surgery (25 patients)), a median number of eight lifetime AEDs prescribed, and $36 \%$ of patients with ten or more lifetime AEDs prescribed (Table 1). A median of three concomitant AEDs were used at brivaracetam initiation. The most frequently prescribed lifetime AED (in combination or monotherapy) was levetiracetam (95.4\% of patients), followed by valproic acid $(84.6 \%)$, lamotrigine $(72.6 \%)$, and carbamazepine

Table 1 Baseline demographic characteristics

\begin{tabular}{ll}
\hline Characteristics & $\begin{array}{l}\text { Real-life data in } \\
\text { Belgium } \\
(N=175)\end{array}$ \\
\hline Gender & \\
Female, $n(\%)$ & $96(54.9)$ \\
Male, $n(\%)$ & $79(45.1)$ \\
Age (years) & \\
Mean (SD) & $38.0(13.4)$ \\
Median (range) & $37.0(16-71)$ \\
Age group & \\
16-25 years, $n(\%)$ & $35(20.0)$ \\
26-45 years, $n(\%)$ & $93(53.1)$ \\
46-65 years, $n(\%)$ & $44(25.1)$ \\
$>65$ years, $n(\%)$ & $3(1.7)$ \\
Non-drug treatment & \\
Yes, $n(\%)$ & $85(48.6)$ \\
No, $n$ (\%) & $90(51.4)$ \\
Distribution of the non-drug treatment $(N=85)$ & \\
Epilepsy surgery, $n(\%)$ & $25(29)$ \\
Vagus nerve stimulation, $n(\%)$ & $72(85)$ \\
Deep brain stimulation, $n(\%)$ & $4(5)$ \\
Atkins diet, $n(\%)$ & $1(1)$ \\
Number of lifetime AEDs & $8.3(3.4)$ \\
Mean (SD) & $8(2-18)$ \\
Median (range) & \\
Number of lifetime AEDs & $45(25.7)$ \\
2-5 AEDs, $n(\%)$ & $89(50.9)$ \\
6-10 AEDs, $n(\%)$ & $41(23.4)$ \\
$>10$ AEDs, $n(\%)$ & \\
\hline
\end{tabular}

$A E D$ antiepileptic drug, $N$ total number of patients included in the analyses, $n / \%$ number/percentage of patients within each category, $S D$ standard deviation
(72.0\%). Among patients with concomitant AED data available at last follow-up, 24/166 (14.5\%) received concomitant levetiracetam.

\subsection{Outcomes}

\subsubsection{Retention}

Percentages of patients still on brivaracetam were $85.8 \%$ (145/169), 73.9\% (119/161), and 64.9\% (85/131) after 3, 6, and 9 months (Fig. 1). Retention rates of brivaracetam using Kaplan-Meier estimates were $88.8 \%, 78.8 \%$, and $69.7 \%$ after 3, 6, and 9 months (Fig. 2). Numbers of censored observations were $6,8,30$, and 85 after the baseline, 3-, 6-, and 9-month visits, respectively. Among the 175 patients included in analyses, 24 (13.7\%) stopped their treatment during the first 3 months, 18 (10.3\%) between 3 and 6 months, and four $(2.3 \%)$ between 6 and 9 months after brivaracetam initiation (Fig. 1).

\subsubsection{Seizure Frequency}

The percentage of patients with fewer than six seizures per month increased from $32.7 \%$ at baseline to $41.3 \%, 42.6 \%$, and $50.7 \%$ after 3, 6, and 9 months, respectively (Fig. 3a). Compared with baseline, seizure frequency decreased in $32.0-37.3 \%$ and remained stable in $49.5-54.1 \%$ of patients after 3-9 months (Fig. 4a).

Among the 126 patients with available information on seizure frequency during the first 3 months of brivaracetam treatment, four (3.2\%) achieved 3-month seizure freedom (Fig. 3a): one ( $0.8 \%)$ remained seizure-free during the entire observation period, one $(0.8 \%)$ until 6 months and was subsequently lost to follow-up, one (0.8\%) was lost to follow-up after 3 months, and one ( $0.8 \%$ ) had seizures between 3 and 6 months after brivaracetam initiation.

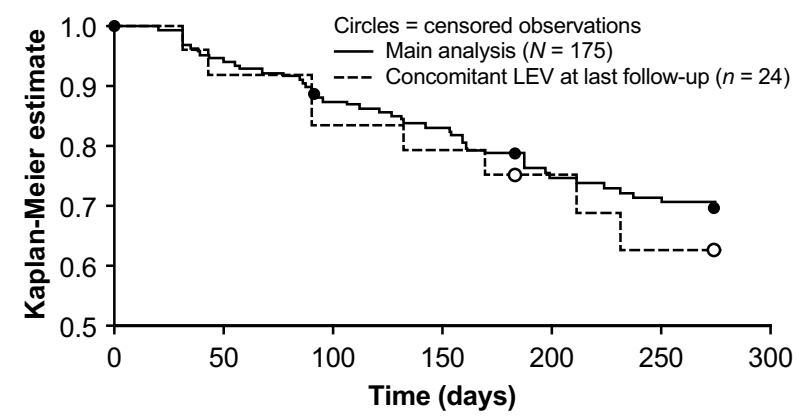

Fig. 2 Retention rate of brivaracetam using the Kaplan-Meier estimate in the 175 patients included in the main analysis and in the 24 patients on concomitant levetiracetam at last follow-up. $L E V$ levetiracetam 

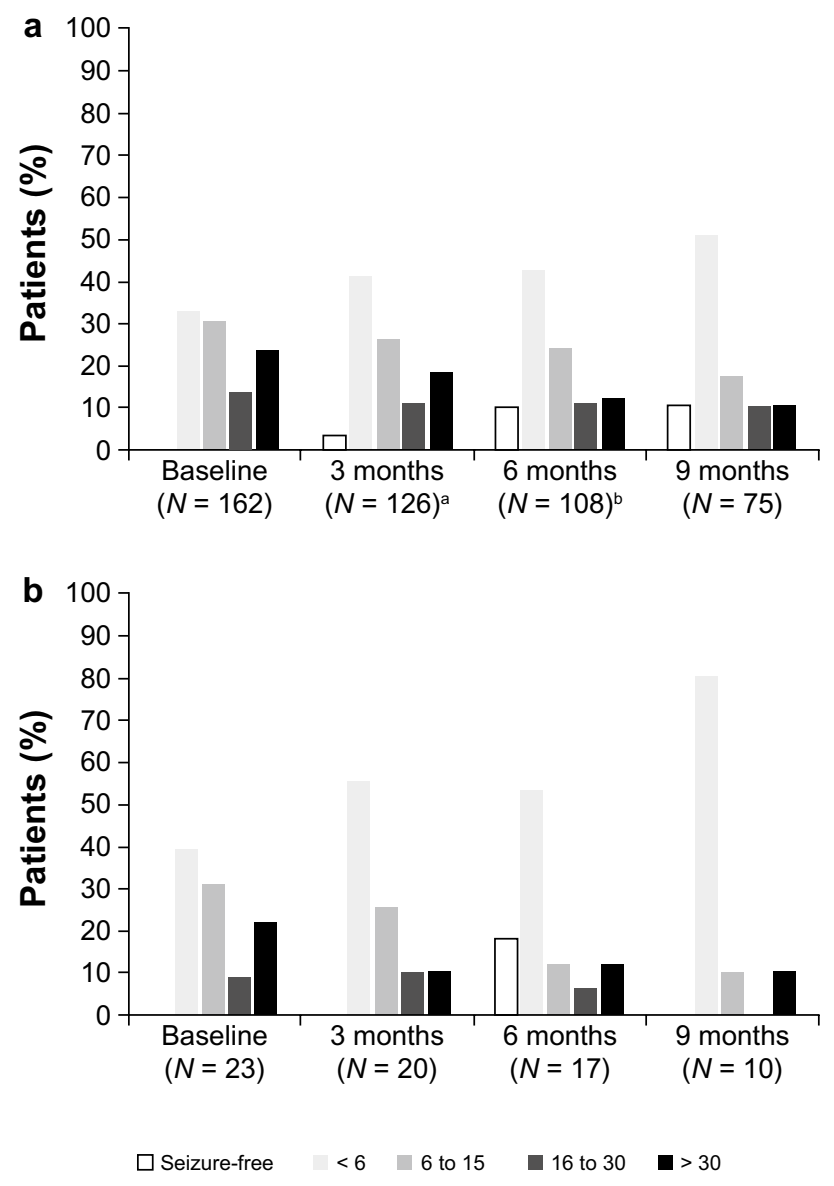

Fig. 3 Percentage of patients according to the monthly seizure frequency reported before treatment initiation and after 3, 6, and 9 months of brivaracetam treatment (a) in all the patients included in the main analyses and (b) in the patients on concomitant levetiracetam at last follow-up. $N$ total number of patients for whom information on quantitative seizure frequency is available, $\%$ percentage of

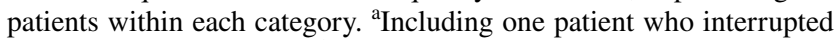

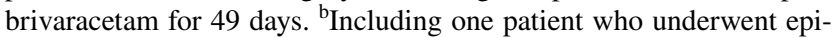
lepsy surgery after start of brivaracetam

Among the 108 patients with available information on seizure frequency between 3 and 6 months after brivaracetam initiation, $11(10.2 \%)$ achieved 3-month seizure freedom (Fig. 3a): six (5.6\%) remained seizure-free between 6 and 9 months, three $(2.8 \%)$ were lost to follow-up after 6 months, and two (1.9\%) had new seizures between 6 and 9 months.

Among the 75 patients with available information on seizure frequency between 6 and 9 months after brivaracetam initiation, eight $(10.7 \%)$ achieved 3-month seizure freedom (Fig. 3a): six (8.0\%) were already registered as seizure-free from previous timepoints and two $(2.7 \%)$ had seizures at previous timepoints.

Six-month seizure freedom was achieved by $7 / 122(5.7 \%)$ patients with available information on seizure frequency for two or more consecutive visits.
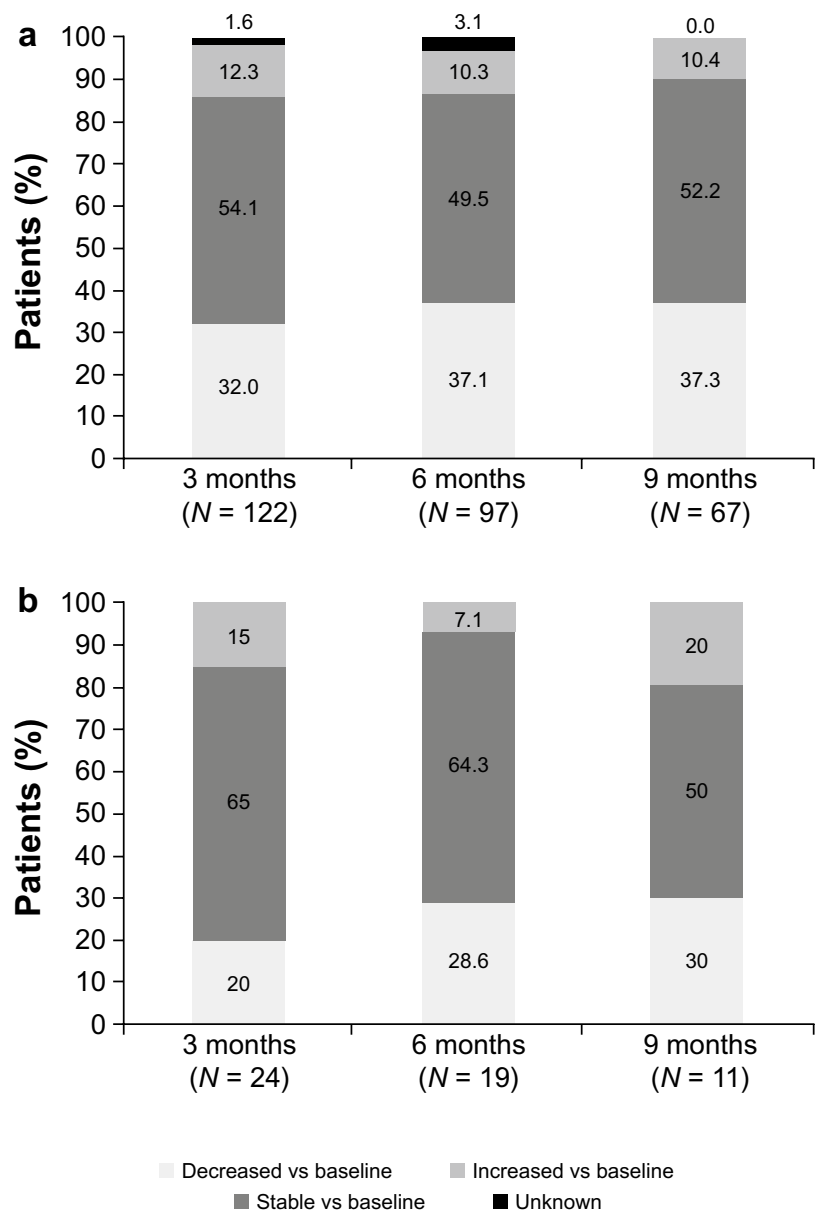

Fig. 4 Monthly seizure frequency evolution after 3, 6, and 9 months of brivaracetam treatment in the patients without seizure freedom (a) included in the main analyses and (b) on concomitant levetiracetam at last follow-up. $N$ total number of patients without seizure freedom at the specified timepoint for whom information on seizure frequency is available at the specified timepoint and at baseline, $\%$ percentage of patients within each category

\subsubsection{Qualitative Seizure Evaluation}

Regarding the qualitative evaluation of seizures by the physicians, 61/138 (44.2\%), 45/116 (38.8\%), and 35/81 (43.2\%) patients had improved, 59/138 (42.8\%), 59/116 (50.9\%), and $41 / 81(50.6 \%)$ patients were considered unchanged, and $18 / 138$ (13.0\%), 12/116 (10.3\%), and 5/81 (6.2\%) patients had worsened at 3,6, and 9 months after treatment start compared with baseline, respectively (Fig. 5a).

\subsubsection{Brivaracetam Treatment Regimen}

The mean number of concomitant AEDs was 2.8 (range 1-7) at brivaracetam initiation and 2.4 (range 1-6) at last followup. Most patients received two or three concomitant AEDs. The concomitant AEDs used were similar at brivaracetam initiation and last follow-up, except for a lower proportion of 

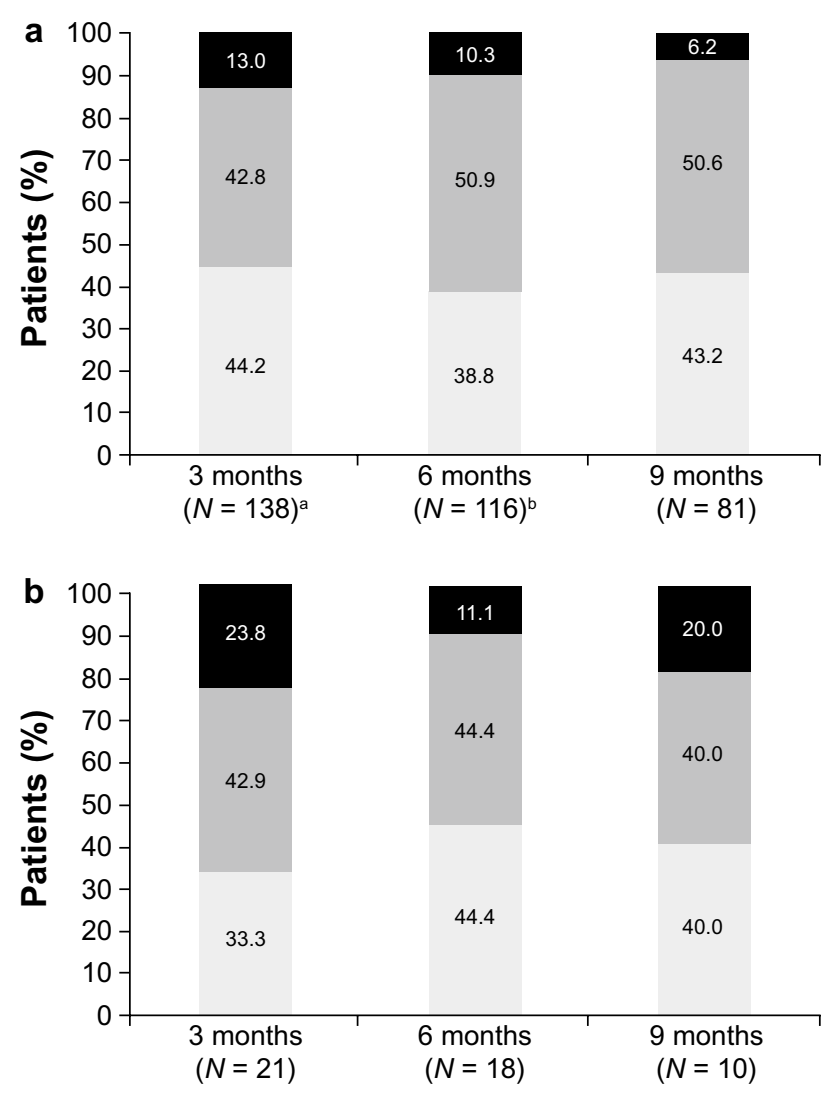

Improvement Stable a Agravation

Fig. 5 Qualitative seizure evaluation after 3, 6, and 9 months of brivaracetam treatment (a) in the patients included in the main analyses and (b) in the patients on concomitant levetiracetam at last followup. $N$ total number of patients for whom information on qualitative seizure frequency is available, \% percentage of patients within each

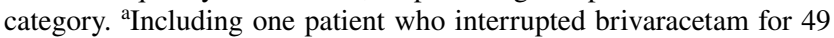
days. ${ }^{b}$ Including one patient who underwent epilepsy surgery after the start of brivaracetam

levetiracetam (14.5\% vs. $33.7 \%)$ and perampanel (7.2\% vs. $11.4 \%$ ) at last follow-up compared with baseline as shown in the table (Online Supplemental Material, Resource 1).

\subsection{Outcomes in the Subpopulation on Concomitant Levetiracetam at Last Follow-Up}

At 3 and 6 months, data were available for all 24 patients on concomitant levetiracetam at last follow-up. At 9 months, data were available for 18 patients due to loss to follow-up for six patients after 6 months.

\subsubsection{Retention}

Percentages of patients remaining on brivaracetam while on concomitant levetiracetam at last follow-up were $79.2 \%$
(19/24 patients), $70.8 \%$ (17/24), and 55.6\% (10/18) after 3, 6, and 9 months, respectively. Retention rates of brivaracetam in patients on concomitant levetiracetam using KaplanMeier estimates were $83.3 \%, 75.0 \%$, and $62.5 \%$ after 3, 6, and 9 months, respectively (Fig. 2). Among the 24 patients included in analyses, five (20.8\%) stopped their brivaracetam treatment during the first 3 months, two (8.3\%) between 3 and 6 months, and one (4.2\%) between 6 and 9 months after brivaracetam initiation.

\subsubsection{Seizure Frequency}

In patients on concomitant levetiracetam at last follow-up, the percentage of patients with fewer than six seizures per month increased from $39.1 \%$ at baseline to $55.0 \%, 52.9 \%$, and $80.0 \%$ after 3, 6, and 9 months of treatment (Fig. 3b). Compared with baseline, seizure frequency decreased in $20-30 \%$ and remained stable in $50-65 \%$ of patients after 3-9 months (Fig. 4b). Three-month seizure freedom was achieved by $3 / 17$ (17.6\%) patients (all between 3 and 6 months after brivaracetam initiation); no patient achieved 6-month seizure freedom (Fig. 3b).

\subsubsection{Qualitative Seizure Evaluation}

Regarding the qualitative evaluation of seizures by the physicians in patients on concomitant levetiracetam at last follow-up, seven (33.3\%), eight (44.4\%), and four (40.0\%) patients had improved, nine (42.9\%), eight (44.4\%), and four (40.0\%) patients were considered stable, and five (23.8\%), two $(11.1 \%)$, and two $(20.0 \%)$ patients had worsened at 3 , 6 , and 9 months after treatment initiation compared with baseline, respectively (Fig. 5b).

\subsubsection{Brivaracetam Treatment Regimen}

In patients on levetiracetam at last follow-up, the mean number of concomitant AEDs prescribed in addition to brivaracetam was 2.9 (range 1-5) at treatment start and last follow-up visit. Most patients received between two and four concomitant AEDs in addition to brivaracetam.

\subsection{Safety}

Four patients reported severe safety events: one reported suicidal thoughts, hetero aggression, and increased seizure frequency; one reported suicidal thoughts; one reported exacerbation of seizures, decreased activity, and loss of appetite; and one reported headache.

The most frequent psychiatric safety events were aggression (15 events) and mood change (depression) (five events). The most common central nervous system-related safety events were dizziness and headache (five events each). The 
most common other safety events were thrombocytopenia (six events), loss of appetite, and nausea (three events each) as shown in Online Supplemental Material (Resource 2).

\section{Discussion}

In this chart review based on data from the MNP in Belgium, we evaluated retention rate and seizure outcomes following brivaracetam as adjunctive therapy for the treatment of focal seizures with or without secondary generalization. The vast majority $(95.4 \%)$ of patients evaluated had been treated previously or concomitantly with levetiracetam. These real-life data are useful to evaluate brivaracetam utility in a broader population than patients enrolled in clinical trials, who are often not representative of everyday clinical practice because of strict inclusion and exclusion criteria [16]. The study population was highly drug resistant, with a median of eight previously used AEDs and three concomitant AEDs at treatment initiation. In addition, almost half of the total study population $(48.6 \%)$ had previously received unsuccessful non-drug treatments, including epilepsy surgery (14.3\% of total population), vagus nerve stimulation ( $41.1 \%$ of total population), and deep brain stimulation (2.3\% of total population).

In these drug-resistant patients, retention rates of brivaracetam, used as indicators of effectiveness and tolerability, were high, with $85.8 \%, 73.9 \%$, and $64.9 \%$ of patients still on brivaracetam after 3,6 , and 9 months. Our results are in line with other published retrospective data showing that brivaracetam is effective in difficult-to-treat epilepsy patients [17-21], including those who have undergone unsuccessful epilepsy surgery or neurostimulation $[17,19]$. In two retrospective studies in Germany, retention rates at 6 months were $75.8 \%$ in patients who failed a median of four previous AEDs (post-marketing study) [19] and $80.4 \%$ in patients with a median of five previously used AEDs [17]. In another retrospective study in Spain, retention rates were $80.2 \%$ at 6 months and $70.4 \%$ at 1 year in patients with a median of eight previously used AEDs [20]. A lower retention rate (51.5\%) was obtained in a monocenter survey in highly intractable patients with a median of ten previously used AEDs in Germany [18]. In another monocenter, retrospective analysis in Germany (median of five previously used AEDs), the retention rate was $72 \%$ during an average period of 5.3 months after brivaracetam initiation [21].

In our chart review, seizure frequency tended to decrease after brivaracetam initiation. Although this is an encouraging finding, these results should be interpreted with caution since patients with inadequate seizure control may have discontinued their treatment before the end of the follow-up (excluded from analyses at subsequent timepoints). Nevertheless, treating physicians also considered that $38.8-44.2 \%$ of patients presented a qualitative seizure improvement and $42.8-50.9 \%$ of patients remained unchanged during the 9-month follow-up. Moreover, $10.2 \%$ of patients achieved 3 -month seizure freedom after 6 months of treatment, and $5.7 \%$ of patients achieved 6-month seizure freedom at any time. Although these rates are good for highly drug-resistant epilepsy patients given that the probability of achieving seizure freedom diminishes substantially with each subsequent AED regimen [22], they should be interpreted cautiously because they depend on baseline seizure frequencies. Threemonth seizure freedom rates after 6 months of brivaracetam treatment were $17.2 \%$ in a previous retrospective study in Spain (median of eight previous AEDs) [20], 15.3\% in a retrospective cohort study in Germany (median of four previous AEDs) [19], and 7.0\% in a monocenter survey in Germany (median of ten previous AEDs) [18]. In a monocenter study in Germany, $21.7 \%$ of patients became newly seizurefree after 6 months (median of five previous AEDs) [17]. In another retrospective study in Germany, the seizure freedom rate was $8.8 \%$ during an average period of 5.3 months after brivaracetam initiation (median of five previous AEDs) [21].

Four patients reported severe safety events, including suicidal thoughts $(n=2)$, aggression $(n=1)$, decreased activity $(n=1)$, loss of appetite $(n=1)$, and headache ( $n$ $=1)$. Besides these, the most frequently reported safety events were aggression (15 events), thrombocytopenia (six events), mood change (depression) (five events), dizziness (five events), and headache (five events). These safety events are generally in line with previous reports, although the most common safety events in the previously mentioned studies also included somnolence, irritability, fatigue, cognitive decline, and nausea [17-21].

The results of this chart review suggest that brivaracetam may be a suitable treatment option for drug-resistant epilepsy patients previously or concomitantly treated with levetiracetam. Indeed, $95.4 \%$ of patients in this chart review were previously or concomitantly treated with levetiracetam (as per eligibility criteria). The good retention and seizure freedom rates observed up to 9 months post-brivaracetam initiation are in line with a previous analysis showing that intolerability or ineffectiveness of prior levetiracetam treatment did not preclude a good response to brivaracetam [17]. In the previous study, the incidence of psychiatric and behavioral problems was lower in patients who switched from levetiracetam to brivaracetam [17]. A potential explanation is that levetiracetam inhibits AMPA receptors, an effect that has been associated with behavioral events for some drugs, whereas brivaracetam does not [23, 24]. Based on our results in the subpopulation of patients on levetiracetam at the last follow-up visit, the combination of these two drugs did not appear to be detrimental: the retention rate at 6 months was $70.8 \%$, and the proportion of patients who reported fewer than six seizures per month increased from 
$39.1 \%$ at baseline to $55.0 \%, 52.9 \%$, and $80.0 \%$ after 3,6 , and 9 months of treatment.

The strengths of this retrospective chart review include its follow-up duration, and the systematic and uniform data collection. Nevertheless, the results should be interpreted cautiously because of the limitations associated with its retrospective design: some data were missing from patient records, such as the exact numbers and types of seizures, and the reasons for previous levetiracetam treatment failures (therapeutic failure and/or adverse effects). A further limitation of the study is that the reason for treatment discontinuation was not collected, nor were brivaracetam doses (although it should be noted that the analysis only included patients using brivaracetam on-label). Furthermore, the high proportion of patients in our study sample previously or concomitantly treated with levetiracetam is not representative of general clinical practice. The safety evaluation was beyond the scope of this chart review, and therefore, safety events reported are most likely incomplete and probably only include those of greatest severity. Finally, the results for the subpopulation of patients on levetiracetam at the last followup visit should be interpreted with caution due to the low number of patients included in the subanalysis.

\section{Conclusions}

In our highly drug-resistant population that predominantly included patients treated previously or concomitantly with levetiracetam, retention rate was high during 9 months after brivaracetam treatment initiation. Quantitative and qualitative seizure improvements were also observed: around $10 \%$ achieved 3-month seizure freedom after 6 and 9 months of treatment, and qualitative seizure improvements were reported in around $40 \%$ of patients. The results suggest that brivaracetam adjunctive therapy may be a suitable treatment option in patients with drug-resistant epilepsy, including those with previous or concomitant levetiracetam treatment.

Supplementary Information The online version contains supplementary material available at https://doi.org/10.1007/s40801-021-00246-7.

Acknowledgements The authors thank the physicians from the six centers who participated in the data collection: Prof. Lieven Lagae (Refractory Epilepsy Center, University Hospitals Leuven), Prof. Susana Ferrao Santos (Centre Hospitalier Neurologique William Lennox, Ottignies and Cliniques Universitaires Saint-Luc, Brussels), Dr. Pascal Vrielynck, Dr. Françoise Liénard (Centre Hospitalier Neurologique William Lennox, Ottignies), Dr. Henri Hauman (Centrum voor Epilepsie en Psycho-Organische Stoornissen, Duffel), Prof. Bernard Sadzot, Dr. Estelle Rikir (Centre Hospitalier Universitaire de Liège), Dr. Benjamin Legros (Hôpital Erasme, Brussels), Dr. Riëm El Tahry (Cliniques Universitaires Saint-Luc, Brussels). The authors thank Claire Verbelen, PhD (Modis, Wavre, Belgium) and Nicole Meinel, PhD (Evidence Scientific Solutions, London, UK) for writing assistance, which was funded by UCB Pharma.

\section{Declarations}

Funding The Medical Need Program as well as this retrospective data collection and related publications were sponsored by UCB Pharma.

Conflicts of Interest CD received grants paid to the institution from UCB Pharma during the conduct of the study as well as grants, speaker's fees, and conference support from UCB Pharma outside the submitted work. WVP received nonfinancial support from UCB Pharma during the conduct of the study as well as personal fees and nonfinancial support from UCB Pharma, grants from EIT Health, and personal fees from Idorsia outside the submitted work. KvR has no relevant relationships to disclose. IL and FF are employees of UCB Pharma.

Ethics Approval The Ethics Committees of all participating centers were approached for their consent of the MNP and to inform them of the data collection. Anonymized data entry was conducted by site personnel or data entry assistants upon request of the treating physicians and approval by Ethics Committees.

Consent to Participate/Consent for Publication In the context of this real-life data collection, no informed consent was required due to the retrospective design and the nature of the information collected. The sponsor only had access to fully anonymized data.

Data Availability The datasets generated and/or analyzed during the current study are not publicly available as data from non-interventional studies is outside of UCB's data sharing policy.

Code availability Not applicable.

Author Contributions FF and IL were responsible for the study conception. CD, WVP, and KvR contributed to the study conception, were participating investigators in the data collection, and members of the Medical Need Program. All authors were responsible for data interpretation. Medical writing assistance (funded by UCB Pharma) was provided under the direction of the authors. All authors reviewed and commented on previous versions of the manuscript. All authors read and approved the final manuscript and agreed to the submission to Drugs-Real World Outcomes.

Open Access This article is licensed under a Creative Commons Attribution-NonCommercial 4.0 International License, which permits any non-commercial use, sharing, adaptation, distribution and reproduction in any medium or format, as long as you give appropriate credit to the original author(s) and the source, provide a link to the Creative Commons licence, and indicate if changes were made. The images or other third party material in this article are included in the article's Creative Commons licence, unless indicated otherwise in a credit line to the material. If material is not included in the article's Creative Commons licence and your intended use is not permitted by statutory regulation or exceeds the permitted use, you will need to obtain permission directly from the copyright holder. To view a copy of this licence, visit $\mathrm{http} / / /$ creativecommons.org/licenses/by-nc/4.0/.

\section{References}

1. Kwan P, Arzimanoglou A, Berg AT, Brodie MJ, Allen Hauser W, Mathern G, et al. Definition of drug resistant epilepsy: consensus proposal by the ad hoc Task Force of the ILAE Commission on Therapeutic Strategies. Epilepsia. 2010;51:1069-77. 
2. Kwan P, Brodie MJ. Early identification of refractory epilepsy. N Engl J Med. 2000;342:314-9.

3. Schmidt D, Schachter SC. Drug treatment of epilepsy in adults. BMJ. 2014;348:g254.

4. Ventola CL. Epilepsy management: newer agents, unmet needs, and future treatment strategies. P T. 2014;39:776-92.

5. Klitgaard H, Matagne A, Nicolas JM, Gillard M, Lamberty Y, De Ryck M, et al. Brivaracetam: rationale for discovery and preclinical profile of a selective SV2A ligand for epilepsy treatment. Epilepsia. 2016;57:538-48.

6. Stephen LJ, Brodie MJ. Brivaracetam: a novel antiepileptic drug for focal-onset seizures. Ther Adv Neurol Disord. 2018;11:1756285617742081.

7. Matagne A, Margineanu DG, Kenda B, Michel P, Klitgaard H. Anti-convulsive and anti-epileptic properties of brivaracetam (ucb 34714), a high-affinity ligand for the synaptic vesicle protein, SV2A. Br J Pharmacol. 2008;154:1662-71.

8. Gillard M, Fuks B, Leclercq K, Matagne A. Binding characteristics of brivaracetam, a selective, high affinity SV2A ligand in rat, mouse and human brain: relationship to anti-convulsant properties. Eur J Pharmacol. 2011;664:36-44.

9. Feyissa AM. Brivaracetam in the treatment of epilepsy: a review of clinical trial data. Neuropsychiatr Dis Treat. 2019;15:2587-600.

10. Yates SL, Fakhoury T, Liang W, Eckhardt K, Borghs S, D’Souza J. An open-label, prospective, exploratory study of patients with epilepsy switching from levetiracetam to brivaracetam. Epilepsy Behav. 2015;52:165-8.

11. European Medicines Agency (EMA). Committee for Medicinal Products for Human Use (CHMP). Summary of opinion (post authorisation)—Briviact (brivaracetam). 2018. https://www.ema. europa.eu/documents/smop/chmp-post-authorisation-summarypositive-opinion-briviact_en.pdf. Accessed 12 Feb 2019.

12. Zaccara G. Brivaracetam: new compound approved for the treatment of epilepsy. Drugs Today (Barc). 2016;52:219-27.

13. European Medicines Agency (EMA). Summary of product characteristics-Briviact. https://www.ema.europa.eu/en/documents/ product-information/briviact-epar-product-information_en.pdf. Accessed 19 Aug 2019.

14. Federal Agency for Medicines and Health Products. Compassionate use-medical need-Briviact—summarized information for publication. https://www.famhp.be/sites/default/files/content/ annex_i_summarized_information_for_publication_en_fr_nl_5. pdf. Accessed 29 Aug 2019.
15. Institut national d'assurance maladie-invalidité (INAMI). Autorisations - formulaires de demande réglementaires-Formulaire de demande de remboursement de la spécialité BRIVIACT en tant que thérapie d'association. 2019. https://ondpanon.riziv.fgov.be/ SSPWebApplicationPublic/fr/Public/RequestForm. Accessed 4 Feb 2019.

16. Blonde L, Khunti K, Harris SB, Meizinger C, Skolnik NS. Interpretation and impact of real-world clinical data for the practicing clinician. Adv Ther. 2018;35:1763-74.

17. Hirsch M, Hintz M, Specht A, Schulze-Bonhage A. Tolerability, efficacy and retention rate of brivaracetam in patients previously treated with levetiracetam: a monocenter retrospective outcome analysis. Seizure. 2018;61:98-103.

18. Steinhoff BJ, Bacher M, Bucurenciu I, Hillenbrand B, Intravooth $\mathrm{T}$, Kornmeier R, et al. Real-life experience with brivaracetam in 101 patients with difficult-to-treat epilepsy: a monocenter survey. Seizure. 2017;48:11-4.

19. Steinig I, von Podewils F, Moddel G, Bauer S, Klein KM, Paule E, et al. Postmarketing experience with brivaracetam in the treatment of epilepsies: a multicenter cohort study from Germany. Epilepsia. 2017;58:1208-16.

20. Villanueva V, Lopez-Gonzalez FJ, Mauri JA, Rodriguez-Uranga J, Olive-Gadea M, Montoya J, et al. BRIVA-LIFE: a multicenter retrospective study of the long-term use of brivaracetam in clinical practice. Acta Neurol Scand. 2018;139:360-8.

21. Zahnert F, Krause K, Immisch I, Habermehl L, Gorny I, Chmielewska I, et al. Brivaracetam in the treatment of patients with epilepsy-first clinical experiences. Front Neurol. 2018;9:38.

22. Chen Z, Brodie MJ, Liew D, Kwan P. Treatment outcomes in patients with newly diagnosed epilepsy treated with established and new antiepileptic drugs: a 30-year longitudinal cohort study. JAMA Neurol. 2018;75:279-86.

23. Coppola G, Iapadre G, Operto FF, Verrotti A. New developments in the management of partial-onset epilepsy: role of brivaracetam. Drug Des Devel Ther. 2017;11:643-57.

24. Hansen CC, Ljung H, Brodtkorb E, Reimers A. Mechanisms underlying aggressive behavior induced by antiepileptic drugs: focus on topiramate, levetiracetam, and perampanel. Behav Neurol. 2018;2018:2064027. 